\title{
PROBADORES DE MAÍZ PARA FACTORES DE INCOMPATIBILIDAD GAMETOFÍTICA
}

\author{
MAIZE TESTERS FOR GAMETOPHYTIC CROSS-INCOMPATIBILITY FACTORS
}

\author{
Lino De la Cruz Larios* ${ }^{1 *}$ J. Jesús Sánchez González ${ }^{1}$, José Ron Parra1, Fernando Santacruz Ruvalcaba ${ }^{1}$, \\ Eduardo Rodríguez Guzmán ${ }^{1}$, José A. Ruíz Corral' ${ }^{2}$ y Moisés M. Morales Rivera ${ }^{1}$
}

\begin{abstract}
${ }^{1}$ Instituto de Manejo y Aprovechamiento de Recursos Fitogenéticos (IMAREFI), Centro Universitario de Ciencias Biológicas y Agropecuarias (CUCBA), Universidad de Guadalajar. Km 15.5 Carretera Guadalajara-Nogales. Apartado Postal 129. 45110. Las Agujas, Nextipac, Mpio de Zapopan, Jalisco, México. Tel. y Fax: 33368207 43. ${ }^{2}$ Centro de Investigación Regional del Pacífico Centro, Instituto Nacional de Investigaciones Forestales, Agrícolas y Pecuarias (INIFAP). Parque Los Colomos S/N. 44660, Guadalajara, Jalisco, México.
\end{abstract}

*Autor para correspondencia (linocucba@hotmail.com)

\section{RESUMEN}

En este trabajo se analizó el comportamiento de líneas de maíz (Zea mays L.) con diferente origen y sus cruzas como probadores, en el estudio de dos sistemas de incompatibilidad en maíz: el factor gametofítico-1 (ga1) y Teosinte crossing barrier-1 (tcb1). Los trabajos se hicieron de 2003 a 2006 en Zapopan, Jalisco; en Las Garzas, Municipio de Guachinango, Jalisco; y en Tapachula, Nayarit, todos ubicados en México. Se usaron siete líneas subtropicales adaptadas, tres accesiones de maíz con composición alélica conocida, cinco híbridos comerciales de empresas privadas, y once líneas de EE. UU. Los genotipos se agruparon en tres experimentos para el estudio de los dos sistemas de incompatibilidad. Los datos de llenado de grano se analizaron con base en los procedimientos GLM y MIXED del Sistema de Análisis Estadístico (SAS). Se identificaron dos cruzas sim-

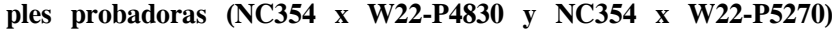
homocigotas para el factor gametofítico-1 (ga1) con el alelo Ga1-S, con capacidad de discriminación y estabilidad. Las accesiones de las razas Maíz Dulce, Dulcillo del Noroeste y Reventador con alelos GaI-S permitieron identificar probadores para el factor gametofitico1. Ninguna de las cruzas simples entre líneas adaptadas y líneas templadas con el alelo $T c b 1-S$, fueron útiles como probadores para el Complejo de Incompatibilidad del Teocintle.

Palabras clave: Zea mays, teocintle, incompatibilidad cruzada.

\section{SUMMARY}

In this study we analized the performance of maize (Zea mays L.) inbred lines from different origins, and their crosses as testers, of two cross-incompatibility systems in maize: the gametophyte factor-1 (gal) and the Teosinte crossing barrier-1 (tcb1). Field experiments were conducted from 2003 to 2006 at Zapopan, Jalisco; at Las Garzas, in Guachinango, Jalisco; and at Tapachula, Nayarit, all of them in México. Seven adapted subtropical inbred lines, three maize accessions with known allelic composition, five commercial hybrids from private institutions, and eleven inbred lines from USA were used. Genetic materials were grouped into three experiments to study the two cross-incompatibility systems. Seed set data was analyzed based on procedures GLM and MIXED of the Statistical Analysis System (SAS). Two single crosses homozygous for Ga1-S were identified as testers for the gametophyte factor-1 (NC354 $\mathrm{x}$
W22-P4830 and NC354 x W22-P5270); these crosses proved to have good stability and discrimination capacity. Accessions of the races Maíz Dulce, Dulcillo del Noroeste and Reventador, with Gal-S allele, allowed the identification of testers for the gametophyte factor-1. No single crosses among adapted and temperate lines with the Tcb1$S$ allele were useful as testers for the Teosinte Incompatibility Complex .

Index words: Zea mays, teosinte, cross-incompatibility.

\section{INTRODUCCIÓN}

El flujo genético entre especies y variedades en áreas naturales y en los campos de los agricultores depende de varios mecanismos de aislamiento, que incluyen distancia y barreras geográficas, sincronía en floración y sistemas de incompatibilidad. La incompatibilidad entre especies o variedades es uno de los mecanismos más efectivos que limitan el flujo genético. Nelson (1996) presentó un informe completo de los factores de incompatibilidad en maíz (Zea mays L.), a los que se refirieron colectivamente como factores gametofiticos. Los factores de incompatibilidad son numerosos en maíz y se han reportado desde la década de 1920 (en White Rice Popcorn). El locus gal es el más estudiado pues el alelo $G a 1-S$ causa esterilidad no-recíproca. Una planta $G a 1-S / G a 1-S$ no acepta polen $g a 1$; sin embargo, el polen $G a 1-S$ de una planta $G a 1-S$ /Gal-S causa un llenado de grano completo en la mazorca de plantas gal/gal. El tercer alelo Ga1-m se ha definido como neutral desde el punto de vista de compatibilidad dado que fertiliza todos los genotipos incluyendo a $G a l-S / G a l-S$ y acepta polen de los tres alelos. Este alelo Gal-m se ha designado como referencia a su acción por medio del gameto masculino.

El Dr. Jerry Kermicle ha investigado la incompatibilidad entre el teocintle y el maíz, y reportado varios factores 
genéticos que limitan el cruzamiento entre ambas especies (Kermicle y Allen, 1990; Kermicle, 1997; Evans y Kermicle, 2001; Kermicle et al., 2006; Kermicle, 2006). Cuando se polinizan estigmas de maíz con polen de teocintle se obtienen progenies híbridas sin dificultad alguna; sin embargo, las cruzas recíprocas, polen de maíz a estigmas de teocintle, no siempre tienen éxito. Por ejemplo, plantas del teocintle Zea mays ssp. mexicana (teocintle tipo maleza del Valle de México y de El Bajío) polinizadas con maíz presentan un mal llenado de grano y en ocasiones no hay producción de grano. Sin embargo, cuando plantas de teocintle Zea mays ssp. parviglumis (teocintle de las partes bajas de México que crece predominantemente silvestre) se polinizan con maíz, el llenado de grano es por lo general abundante. Evans y Kermicle (2001) describieron el locus Teosinte crossing barrier-1 (tcb1), responsable del reconocimiento entre el polen y los estigmas, el cual "podría estar jugando un papel muy importante en el aislamiento reproductivo entre el maíz y el teocintle". De manera similar que en el locus gal, en este sistema existe el alelo $T c b 1-S$, el cual es completamente dominante sobre $t c b 1$ y un tercer alelo, $T c b 1-m$, con comportamiento neutral. Adicionalmente, para que $T c b 1-S$ manifieste una barrera efectiva en los estigmas, se requiere la acción estabilizadora de un gene modificador ligado a $T c b 1$ y Gal (Evans y Kermicle, 2001).

La mayor parte de los estudios relacionados con la elección de probadores apropiados se han enfocado a evaluar el potencial de rendimiento de líneas de maíz. Matzinger (1953) define como "probador deseable" aquel que combina la simplicidad en su uso con la máxima información en el comportamiento de las líneas. La prueba de progenie fue definida por Allard, citado por Hallauer y Miranda (1988), como "una prueba del valor de un genotipo basada en el comportamiento de su progenie producida en algún sistema de apareamiento definido". Existen diferentes definiciones acerca de los probadores. Rawlings y Thompson (1962) definieron un "buen probador" al que clasifica correctamente el comportamiento de las líneas y discrimina eficientemente entre las líneas bajo prueba. Hallauer (1975) y Hallauer y Miranda (1988) revisaron los aspectos teóricos y evidencias experimentales para determinar la etapa apropiada para llevar a cabo las pruebas de las líneas y otros aspectos relevantes que definen la elección de los probadores; ellos indican que el tipo de acción génica es una de las variables más importantes para definir el probador apropiado; en general sugieren el uso de líneas puras élite para evaluar aptitud combinatoria.

Para desarrollar variedades comerciales que requieran aislamiento de tipos diferentes, así como en estudios de evaluación del flujo genético entre poblaciones, es necesario disponer de una adecuada caracterización genética y fisioló- gica de los sistemas de incompatibilidad. Sin embargo, para caracterizar apropiadamente a las especies del género Zea, es indispensable contar con probadores apropiados que permitan clasificar de manera correcta y fácil, a cualquier material de interés para el carácter de incompatibilidad.

Los sistemas conocidos en maíz que causan incompatibilidad entre polen y estigmas se heredan de manera Mendeliana simple; sin embargo, el alelo Gal-S es parcialmente dominante sobre gal mientras que el alelo $T c b 1-S$ es completamente dominante sobre $t c b 1$. Desde el punto de vista de caracterización de materiales, los probadores con el alelo Gal-S deben ser homocigotos mientras que los probadores con $T c b 1-S$ pueden ser homocigotos o heterocigotos. Esta consideración en la elección de los probadores pudiera resultar sencilla; sin embargo, se ha manifestado la presencia de genes modificadores de la incompatibilidad que pueden inhibir o activar el efecto de Gal-S o de Tcb1-S (Nelson, 1952; Evans y Kermicle, 2001; Kermicle, 2006).

Con base en lo anterior, el presente trabajo tuvo como objetivo estudiar el comportamiento de líneas de maíz con diferente origen y sus cruzas, como probadores en el estudio de sistemas de incompatibilidad en especies del género Zea.

\section{MATERIALES Y MÉTODOS}

El estudio se efectuó en el Centro Universitario de Ciencias Biológicas y Agropecuarias (CUCBA) de la Universidad de Guadalajara (UDG), ubicado en Zapopan, Jalisco; en la localidad de las Garzas del municipio de Guachinango, Jalisco; y en el Centro de Investigaciones de la empresa Pioneer Hi-Bred International en Tapachula, Nayarit, México. El CUCBA está situado en Nextipac, a $20^{\circ}$ $45^{\prime} \mathrm{LN}$ y $103^{\circ} 31^{\prime} \mathrm{LO}$, con una elevación de $1650 \mathrm{~m}$; su temperatura media durante junio-octubre es de $23.6{ }^{\circ} \mathrm{C}$ y su precipitación media anual es de $816 \mathrm{~mm}$. Las Garzas está situado a $20^{\circ} 47^{\prime} \mathrm{LN}$ y $104^{\circ} 24^{\prime} \mathrm{LO}$, con una elevación de $760 \mathrm{~m}$, temperatura media de diciembre a junio de $24.8{ }^{\circ} \mathrm{C}$ y por lo general no hay lluvias en dicho periodo. Tapachula está situada a $20^{\circ} 49^{\prime} \mathrm{LN}$ y $105^{\circ} 55^{\prime} \mathrm{LO}$, con una elevación de $26 \mathrm{~m}$; su temperatura media durante el periodo de enero-junio es de $25{ }^{\circ} \mathrm{C}$ en el que por lo general no llueve. Las siembras experimentales se establecieron en el ciclo de otoño-invierno 2004 en Tapachula, en los ciclos primavera-verano de 2003, 2004 y 2005 en Zapopan y en el ciclo otoño-invierno de 2006 en Las Garzas.

Se usaron genotipos adaptados a las condiciones del ciclo primavera-verano en Zapopan, provenientes de la UDG, del Centro Internacional de Mejoramiento de Maíz 
y Trigo (CIMMYT) y de cuatro empresas privadas. Además, se usaron líneas de origen tropical desarrolladas en la Universidad de Carolina del Norte, Raleigh, EE. UU. donadas por el Dr. M. M. Goodman, y líneas de origen templado provenientes de la Universidad de Wisconsin, Madison, EE. UU. donadas por el Dr. J. Kermicle. En el Cuadro 1 se presenta el origen, tipo de material y composición alélica de los materiales.

En los ciclos de otoño-invierno 2004 en Tapachula, y de primavera-verano 2003, 2004 y 2005 en Zapopan, se sembraron todos los materiales y se obtuvieron datos de las fechas al inicio y a $50 \%$ de liberación del polen y de aparición de los estigmas. Con base en esa información, se programaron las fechas de siembra apropiadas para constituir los probadores $G a 1-S / G a 1-S$ y $T c b 1-S / t c b 1$, así como para llevar a cabo las pruebas de incompatibilidad y los incrementos de las líneas y probadores.

Los materiales genéticos se emplearon para investigar la constitución genética de líneas adaptadas a las condiciones de Zapopan; e identificar las cruzas simples apropiadas para las pruebas de incompatibilidad asociada al locus gal y al locus tcb1. Con tales propósitos se efectuaron los siguientes experimentos.

\section{Experimento 1}

En la primavera-verano de 2003 en Zapopan, se evaluaron como hembras a seis cruzas simples homocigotas para el alelo $G a 1-S$, obtenidas al cruzar las dos líneas de origen tropical (NC348 y NC354) por tres líneas de origen templado (Hy:1001, W22-P4830 y W22-P5270). Como fuentes de polen se usaron seis materiales con el alelo Ga1-S (Jal-304, Nay-39, Sin-25, Hy:1001, W22P4830 y W22-P5270), cuatro con el alelo Tcb1-S (W22P5236, W22-TIC V 1.7, W22-TIC V2.3-1, W22-TIC V2.3-2), uno con el alelo Tcb1-m (W22-P4031) y dos materiales recesivos para $g a 1$ y $t c b 1$ (W22 y P-3394). Se hicieron cruzamientos manuales de cinco plantas individuales de cada progenitor masculino a cada una de las seis cruzas simples usadas como hembra. Los cruzamientos fueron en forma manual, planta a planta; por lo general, se cubrieron espigas de 7:30 a 9:00 h y las polinizaciones se llevaron a cabo de 12:00 a 14:00 h en estigmas de 5 a $8 \mathrm{~cm}$ de longitud.

Cuadro 1. Materiales empleados para identificar probadores de incompatibilidad en maíz, con su procedencia y loci estudiados.

\begin{tabular}{|c|c|c|c|c|c|}
\hline \multirow{2}{*}{ Material } & \multirow{2}{*}{ Tipo } & \multicolumn{3}{|c|}{ Locus } & \multirow[b]{2}{*}{ Origen $^{\dagger \dagger}$} \\
\hline & & gal & $t c b 1$ & $S t b^{\dagger}$ & \\
\hline LUG-358 & Línea & & & & UDG \\
\hline LUG-14 & Línea & & & & UDG \\
\hline LUG-282 & Línea & & & & UDG \\
\hline LUG-379 & Línea & & & & UDG \\
\hline NC348 & Línea & Ga1-S & $t c b 1$ & & NCSU \\
\hline NC354 & Línea & Ga1-S & $t c b 1$ & & $\mathrm{NCSU}$ \\
\hline W22-P4830 & Línea & Ga1-S & $t c b 1$ & & WIS \\
\hline W22-P5270 & Línea & Gal-S & $t c b 1$ & & WIS \\
\hline Нy: 1001 & Línea & Ga1-S & $t c b 1$ & & WIS \\
\hline W22-P5236 & Línea & gal & $T c b 1-S$ & & WIS \\
\hline $\mathrm{W} 22$ & Línea & gal & $t c b 1$ & & WIS \\
\hline P-3394 & Híbrido & gal & $t c b 1$ & & Pioneer \\
\hline DK-2010 & Híbrido & gal & $t c b 1$ & & Monsanto \\
\hline $\mathrm{Z} 30$ & Híbrido & gal & $t c b 1$ & & Hartz Seed \\
\hline P-3028W & Híbrido & gal & $t c b 1$ & & Pioneer \\
\hline P-30G54 & Híbrido & gal & $t c b 1$ & & Pioneer \\
\hline JAL-304 (Maíz Dulce) & Raza & Gal-S & & & CIMMYT \\
\hline NAY-39 (Reventador) & Raza & Ga1-S & & & CIMMYT \\
\hline SIN-25 (Dulcillo del NO) & Raza & Ga1-S & & & CIMMYT \\
\hline
\end{tabular}

${ }^{\dagger}$ Stb es un gene modificador que estabiliza y activa la acción de Tcb1-S; ${ }^{\dagger \dagger}$ UDG = Universidad de Guadalajara; NCSU = North Carolina State University, Raleigh; WIS = Universidad de Wisconsin, Madison; CIMMYT = Centro Internacional de Mejoramiento de Maíz y Trigo. 


\section{Experimento 2}

Este experimento incluyó siete líneas adaptadas a las condiciones de Zapopan, obtenidas en el programa de mejoramiento de la UDG (LUG-03, LUG-14, LUG-282, LUG-357, LUG-358, LUG-379 y LUG-17L), las cuales se caracterizaron durante los ciclos de verano 2004 y 2005 en el CUCBA con dos tipos de probadores: (i) Como probador macho se usó al híbrido P-3394 (gal/gal) que proporcionó el polen a cada una de las líneas para separar las plantas con los alelos Gal-m o gal (con producción de grano) de aquéllas con el alelo Gal-S (sin producción de grano); (ii) Como probadores hembra (GalS/Gal-S) se emplearon dos líneas (NC348 y NC354) y dos cruzas simples (NC-354 x W22-P4830 y NC-354 x W22-P5270) que recibieron polen de cada una de las siete líneas, para separar las plantas con el alelo Gal-m (con grano) de aquéllas con el alelo gal (sin grano).

\section{Experimento 3}

Esta parte del estudio se hizo en Las Garzas, en otoñoinvierno de 2005-2006, y la evaluación, incluyó como hembras a 20 cruzas simples entre siete líneas de la UDG (LUG-03, LUG-14, LUG-282，LUG-357，LUG-358, LUG-379 y LUG-17L) y tres fuentes de germoplasma con el alelo $T c b 1-S$ y el estabilizador $S t b$ (W22-TIC V 1.7, W22-TIC V2.3-1, W22-TIC V2.3-2). No se tuvo semilla disponible de la cruza LUG17L x W22-TIC V2.3-1. Como fuentes de polen se usaron cuatro híbridos comerciales, DK-2010 (Monsanto), Z30 (Hartz Seed), P-3028W y P-30G54 (Pioneer). Se hicieron cruzamientos manuales de cinco plantas individuales de cada progenitor masculino a cada una de las veinte cruzas simples usadas como hembra.

El llenado de grano en la mazorca se utilizó para definir la utilidad de las líneas y cruzas simples como probadores. La estimación de llenado de grano se llevó a cabo visualmente en cada uno de los cruzamientos indicados en los tres experimentos. Las estimaciones se redondearon a $10 \%$ de aproximación; esto es, al llenado abajo de $5 \%$ se le asignó $0 \%$, de 5 a $15 \%$ el valor fue $10 \%$, y así hasta avanzar a $95 \%$ al cual se asignó $100 \%$. La observación se hizo en el lado con el mejor llenado en toda su longitud; cada mazorca se evaluó individualmente.

Para los análisis de varianza se aplicó el Procedimiento GLM con el paquete SAS (SAS Institute, 1992). En los Experimentos 1 y 3 las fuentes de variación fueron: hembras, machos e interacción. En el Experimento 2 las fuentes de variación fueron: líneas, probadores e interacción. En los casos en que la interacción no resultó significativa, se determinaron las diferencias significativas entre las hembras o entre los machos, con base en el procedimiento MIXED del SAS.

\section{RESULTADOS Y DISCUSIÓN}

En la Figura 1 (fila superior) se muestran las mazorcas polinizadas de P-3394, LUG-14, LUG-03 y LUG-282 respectivamente, por la línea W22 (gal/gal); en la fila central, las mazorcas de las cruzas de las hembras ( $\mathrm{Gal}$ $S / G a 1-S$ ) NC348 y en la fila inferior las de NC354 por las líneas macho W22, LUG-14, LUG-03 y LUG-282. Las mazorcas de la fila superior, con llenado completo, indican que los materiales en prueba tienen una constitución alélica Ga1-m/Gal-m o gal/gal. Las mazorcas de las demás filas indican que LUG-14 tiene un genotipo gal/gal debido al deficiente llenado de mazorca cuando polinizó a NC348 y NC354. Por su parte, LUG-03 y LUG-282 tienen genotipo Gal-m/Gal- $m$ dado que el llenado de mazorca en NC348 y NC354 fue completo. El llenado parcial de grano de 5 a $20 \%$ observado en las cruzas de NC348 con W22 y LUG-14, puede ser el resultado de inhibidores de la acción del alelo $G a 1-S$, en NC348. En cambio, NC354 fue consistente y podría ser una línea útil para estudiar incompatibilidad en especies del género Zea.

\section{Experimento 1}

En el análisis de varianza (Cuadro 2) se detectaron diferencias $(P<0.01)$ entre hembras y entre machos, pero no para la interacción hembras x machos. Estos resultados indican que el llenado de grano entre las cruzas simples hembra $(G a 1-S / G a 1-S)$ fue diferente, así como entre los diferentes machos que involucraron fuentes de polen con diferentes alelos de los loci gal y $t c b 1$. La interacción no significativa indica que cada fuente de polen ocasionó llenados de grano en la misma magnitud en cada una de las seis cruzas simples $(G a 1-S / G a 1-S)$. La hembra NC354 x W22-P4830 (Cuadro 3) mostró el menor porcentaje de llenado de grano, aunque las diferencias entre las seis cruzas simples hembra no fueron superiores a $10 \%$. Los machos mostraron marcadas diferencias en el llenado de las mazorcas, según el alelo involucrado. El alelo Gal-S, independientemente de su origen, causó llenados superiores a $90 \%$; los demás alelos ( $g a 1, T c b 1-m$ y $T c b 1-S)$ tuvieron llenados inferiores a $2 \%$ (iguales estadísticamente a $0 \%$ ). Los testigos Jal-304, Nay-39 y Sin-25 cruzados con las seis cruzas simples hembra mostraron consistencia como machos, con llenados de grano de $100 \%$. El promedio de llenado de mazorca de las cruzas simples hembra $G a 1-S / G a 1-S$ fue $97 \%$ al recibir polen de los machos con el alelo Gal-S, $2 \%$ con polen del alelo Tcbl-m, $0 \%$ al recibir polen con el alelo $T c b 1-S$, y $1 \%$ con polen del alelo recesivo gal (Cuadro 4). 


\section{Experimento 2}

Las líneas adaptadas polinizadas con el probador macho recesivo $\mathrm{P}-3394$ mostraron diferencias $(\mathrm{P}<0.01)$ (Cuadro 2). El porcentaje de llenado de grano de estas líneas hembra al usar el macho P3394 fue menor en LUG$358(48 \%)$ y LUG-17L (76 \%) (Cuadro 5), mientras que en las demás líneas el llenado de grano fue cercano a $100 \%$.

Cuadro 2. Análisis de varianza para los experimentos de incompatibilidad en maíz.

\begin{tabular}{|c|c|c|c|}
\hline Fuente de variación & $\mathrm{gl}$ & $\mathrm{C} \mathrm{M}$ & $\mathrm{Fc}$ \\
\hline \multicolumn{4}{|c|}{$\begin{array}{ll}\text { Experimento } 1 & (\mathrm{CV}=18 \%)\end{array}$} \\
\hline Hembras & 5 & 539 & $7.9^{* *}$ \\
\hline Machos & 12 & 70710 & $1039.6 * *$ \\
\hline Hembras x Machos & 60 & 82 & $1.2 \mathrm{~ns}$ \\
\hline \multicolumn{4}{|c|}{ Experimento 2 (Probador macho P-3394) $(\mathrm{CV}=16 \%)$} \\
\hline Líneas & 6 & 1892 & $9.5^{* *}$ \\
\hline \multicolumn{4}{|c|}{ Experimento 2 (Probadores hembra GaI-S) $(\mathrm{CV}=39 \%)$} \\
\hline Hembras & 3 & 645 & $2.6 \mathrm{~ns}$ \\
\hline Líneas & 6 & 42093 & $170.5^{* *}$ \\
\hline Hembras x Líneas & 16 & 498 & $2.0 *$ \\
\hline \multicolumn{4}{|c|}{ Experimento $3(T c b 1)(\mathrm{CV}=59 \%)$} \\
\hline Hembras & 19 & 14477 & $12.3^{* *}$ \\
\hline Machos & 3 & 1621 & $1.4 \mathrm{~ns}$ \\
\hline Hembras x Machos & 56 & 1697 & $1.4 *$ \\
\hline
\end{tabular}

Cuadro 3. Medias de llenado de grano (\%) para las cruzas simples hembras de maíz con genotipo Ga1-S/Gal-S, probadas con líneas, razas y un híbrido (machos) con diferentes alelos conocidos (Experimento 1).

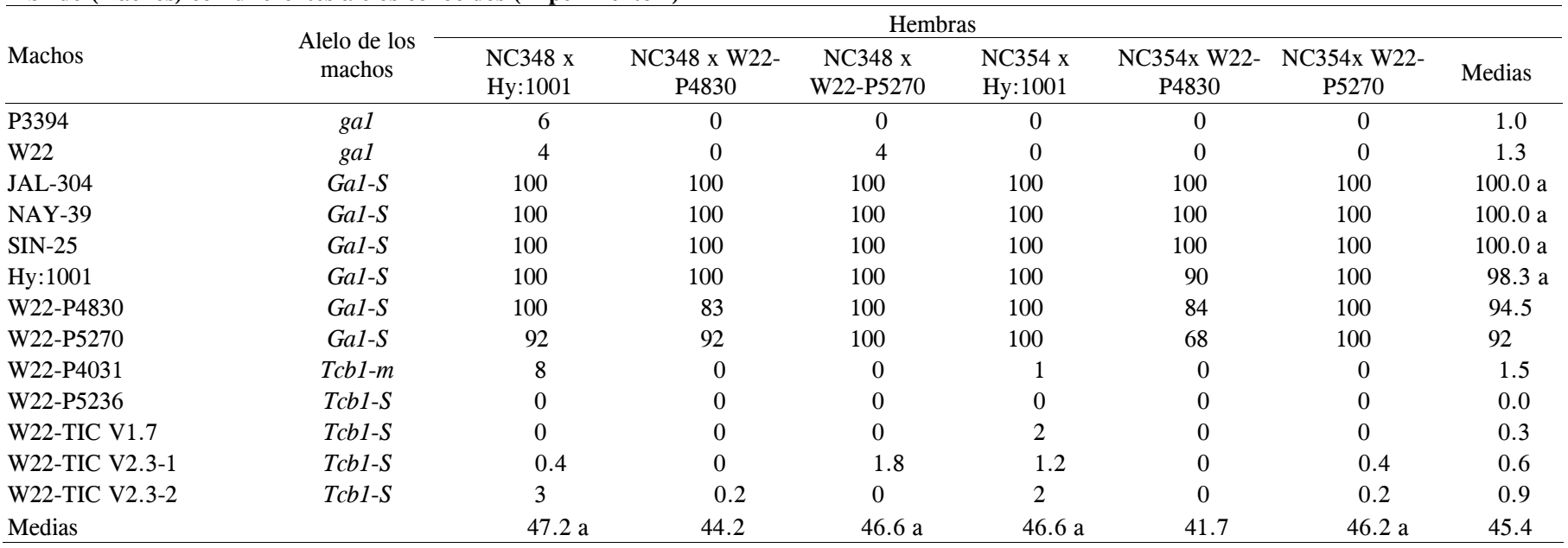

Medias con letras iguales no son estadísticamente diferentes (DMS 0.05). 


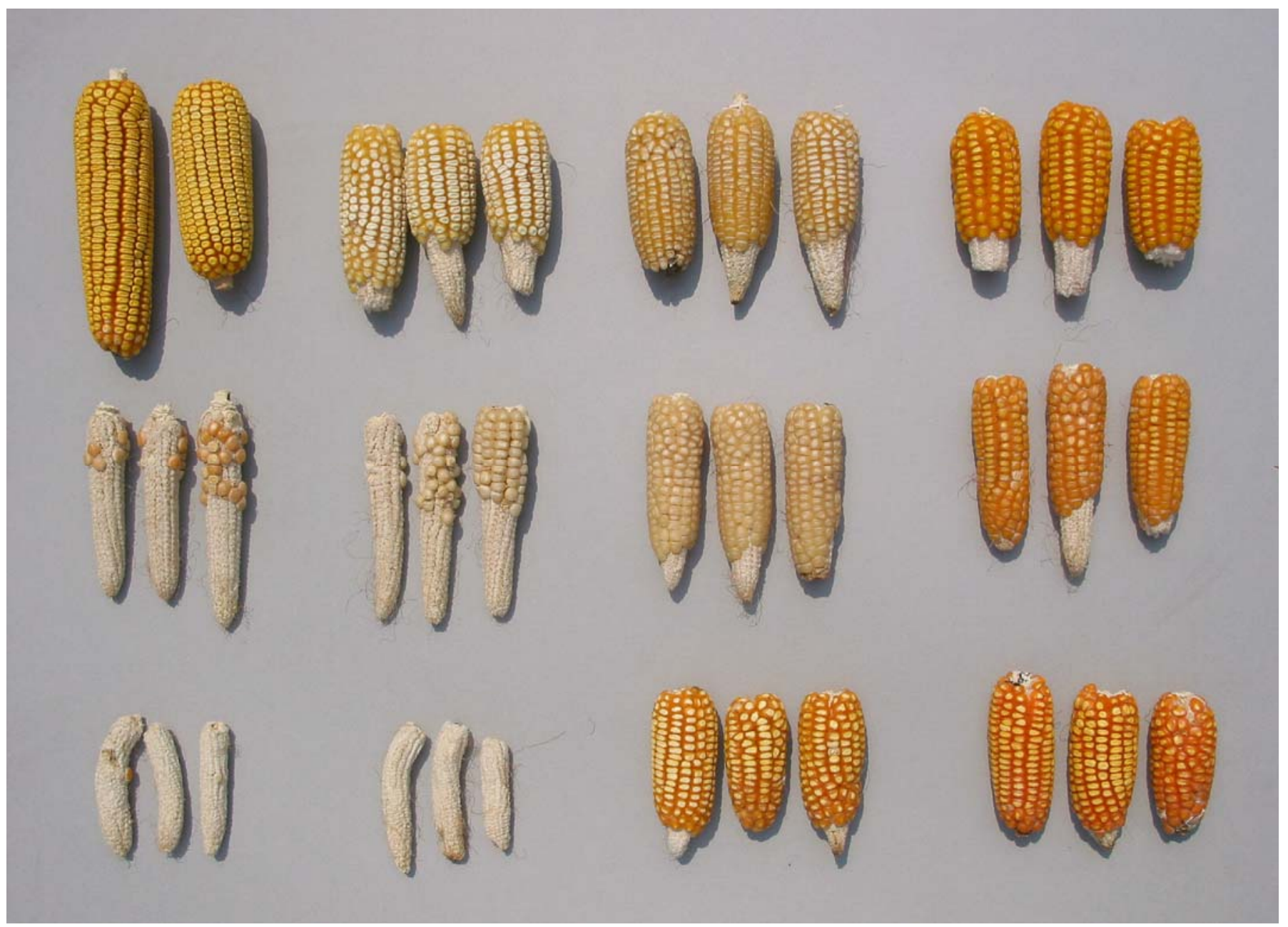

Figura 1. Fila superior de izquierda a derecha: P-3394, LUG-14, LUG-03, LUG-282 (polinizados por W22 ga1/ga1); fila central NC348 Ga1-S/Ga1$S$ (polinizada por W22, LUG-14, LUG-03, LUG-282); fila inferior NC354 Ga1-S/Ga1-S (polinizada por W22, LUG-14, LUG-03, LUG-282).

Cuadro 4. Medias de llenado de grano (\%) para las cruzas simples hembra de maíz con genotipo GaI-S/Gal-S probadas con varios alelos conocidos como macho (Experimento 1).

\begin{tabular}{lccccc}
\hline \multirow{2}{*}{ Hembra } & \multicolumn{5}{c}{ Alelos } \\
\cline { 2 - 6 } & $g a 1$ & Ga1-S & Tcb1- $m$ & Tcb1-S & Media \\
\hline NC348 x Hy:1001 & 5 & 99 & 8 & 1 & 47.2 \\
NC348 x W22-P4830 & 0 & 96 & 0 & 0 & 44.2 \\
NC348 x W22-P5270 & 2 & 100 & 0 & 0 & 46.6 \\
NC354 x Hy:1001 & 0 & 100 & 1 & 1 & 46.6 \\
NC354 x W22-P4830 & 0 & 90 & 0 & 0 & 41.7 \\
NC354 x W22-P5270 & 0 & 100 & 0 & 0 & 46.2 \\
Media & 1 & 97 & 2 & 0 & \\
\hline
\end{tabular}

Cuando las líneas adaptadas se usaron como machos no se detectaron diferencias estadísticas significativas $(\mathrm{P}>$ $0.05)$ entre los probadores hembras $(G a 1-S / G a 1-S)$, pero sí entre líneas $(\mathrm{P}<0.01)$ y para la interacción hembras $\mathrm{x}$ línea $(\mathrm{P}<0.05)$. Las líneas y cruzas simples hembra $(G a 1-S / G a 1-S)$ tuvieron en promedio llenados de mazorca semejantes al ser polinizadas con diferentes líneas adaptadas macho; es decir, tienen una capacidad similar de dis- criminación. De acuerdo con los promedios del porcentaje de llenado (Cuadro 5), la interacción significativa podría atribuirse mayormente a la línea LUG-357 y probablemente en menor cuantía a LUG-282. Con base en estos resultados, la constitución alélica de las líneas es: LUG14, LUG-358, LUG-379 y LUG-17L, recesivas gal/gal; LUG-03 y LUG-282, homocigotas Gal-m/Gal-m; y LUG-357, debido a las diferencias en llenado de grano, parece tener plantas con el alelo gal y plantas con Gal-m, sin descartar la posible presencia de genes que modifican la acción de Gal-S.

\section{Experimento 3}

Se detectaron diferencias $(\mathrm{P}<0.01)$ entre hembras $T c b 1-S / t c b 1$, pero no entre machos (gal/gal, tcb1/tcb1) y sí para la interacción hembras por machos $(\mathrm{P}<0.05)$, lo que indica que las veinte cruzas simples $(T c b 1-S / t c b 1)$ tuvieron llenados de grano en la mazorca de diferente 
magnitud al recibir polen de los probadores macho recesi$\operatorname{vos}($ gal/gal, $t c b 1 / t c b 1)$.

No hubo una tendencia definida en cuanto al comportamiento de las cruzas simples con los machos; sin embargo, se puede decir que las cruzas simples que involucran a las líneas LUG-03 y LUG-17L presentaron los menores porcentajes de llenado (Cuadro 6). Se considera que ninguna de las cruzas simples evaluadas sería apropiada como probador del locus tcbl, dado que no mostraron consistencia en discriminar los materiales machos. Se podría esperar que debido a la existencia de inhibidores de la acción de $T c b 1-S$, se presentaran situaciones de llenado parcial de grano; sin embargo, hubo casos como el de
LUG-358 x W22-TIC V 1.7, en que el llenado fue $100 \%$ con todos los machos; situación similar ocurrió con las cruzas que involucraron a la línea LUG-379.

La elección de probadores apropiados es un tema complejo en el mejoramiento genético de cultivos debido al tipo de herencia de los caracteres en estudio y a las interacciones entre genotipos y ambientes. En los sistemas de incompatibilidad se busca que los probadores involucrados clasifiquen y discriminen correctamente el comportamiento de cualquier tipo de material, como líneas homogéneas, híbridos, poblaciones heterogéneas y especies silvestres; todo esto, aún sin conocer el origen y pedigrí del material.

Cuadro 5. Medias de llenado de grano (\%) para líneas y cruzas simples hembra de maíz con genotipo Ga1-S/Ga1-S y P-3394 como macho con genotipo recesivo gal/gal para probar las líneas adaptadas de la UDG (Experimento 2).

\begin{tabular}{lcrrrr}
\hline Hembra/Macho & Macho & \multicolumn{3}{c}{ Hembras } \\
\cline { 2 - 6 } & P-3394 & NC348 & NC354 & NC354 x W22-P4830 & NC354 x W22-P5270 \\
\hline LUG-03 & $100 \mathrm{a}$ & 100 & 100 & 100 & 0 \\
LUG-14 & $100 \mathrm{a}$ & 1 & 0 & 0 & 0 \\
LUG-282 & $98 \mathrm{a}$ & 86 & 100 & 68 & 100 \\
LUG-357 & $88 \mathrm{a}$ & 60 & 08 & 40 & 99 \\
LUG-358 & 48 & 2 & 0 & 0 & 0 \\
LUG-379 & $100 \mathrm{a}$ & & 0 & 0 & 0 \\
LUG-17L & 76 & 2 & & 0 & 0 \\
\hline
\end{tabular}

Medias con letras iguales no son estadísticamente diferentes (DMS, 0.05).

Cuadro 6. Medias de llenado de grano (\%) para las cruzas simples de maíz con alelo Tcb1-S a probar con híbridos comerciales como macho con genotipo recesivo gal/ga1 (Experimento 3).

\begin{tabular}{|c|c|c|c|c|}
\hline \multirow{2}{*}{ Hembra } & \multicolumn{4}{|c|}{ Machos } \\
\hline & DK-2010 & $\mathrm{Z30}$ & P3028W & P30G54 \\
\hline LUG-357 x W22-TIC V1.7 & 78 & 0 & 100 & 76 \\
\hline LUG-357 x W22-TICV 2.3-1 & 62 & 36 & 28 & 60 \\
\hline LUG-357 x W22-TICV 2.3-2 & 61 & 40 & 80 & 58 \\
\hline LUG-358 x W22-TIC V 1.7 & 100 & 100 & 100 & 100 \\
\hline LUG-358 x W22-TIC V 2.3-1 & 100 & 64 & 80 & 90 \\
\hline LUG-358 x W22-TIC V 2.3-2 & 100 & 71 & & 84 \\
\hline LUG-03 x W22-TIC V1.7 & 32 & 14 & 14 & 10 \\
\hline LUG-03 x W22-TIC V2.3-1 & 28 & 44 & 12 & 2 \\
\hline LUG-03 x W22-TIC V2.3-2 & 26 & 24 & 0 & 4 \\
\hline LUG-14 x W22-TIC V1.7 & 63 & 81 & 42 & 68 \\
\hline LUG-14 x W22-TIC V2.3-1 & 8 & 42 & 45 & 11 \\
\hline LUG-14 x W22-TIC V2.3-2 & 60 & 58 & 32 & 56 \\
\hline LUG-282 x W22-TIC V1.7 & 48 & 71 & 100 & 52 \\
\hline LUG-282 x W22-TIC V2.3-1 & 76 & 40 & 78 & 30 \\
\hline LUG-282 x W22-TIC V2.3-2 & 77 & 44 & 80 & 82 \\
\hline LUG-379 x W22-TIC V1.7 & 100 & 98 & 82 & 100 \\
\hline LUG-379 x W22-TIC V2.3-1 & 100 & 70 & 100 & 100 \\
\hline LUG-379 x W22-TIC V2.3-2 & 56 & 100 & 100 & 100 \\
\hline LUG-17L x W22-TIC V1.7 & 1 & 18 & 20 & 39 \\
\hline LUG-17L x W22-TIC V2.3-2 & 28 & 24 & 40 & 5 \\
\hline
\end{tabular}


En el presente trabajo los resultados de diferentes probadores para el factor gametofitico-1 (gal) son por lo general consistentes, pues muestran un alto grado de estabilidad al discriminar los materiales estudiados. Con base en el comportamiento como hembras de las líneas y cruzas simples homocigotas para $G a 1-S$, se puede deducir que la combinación de NC354 con W22-P4830 y W22P5270 origina los probadores más deseables (Experimento 1). Estos probadores presentan estigmas con una barrera muy efectiva para polen del tipo gal, Tcb1-m y $T c b 1-S$ $S t b$ (locus estabilizador, Stb). Estos resultados se aplicaron en un grupo de siete líneas adaptadas, en las que no se tenía conocimiento de su constitución alélica (Experimento 2). La clasificación de las líneas se hizo con base en el uso simultáneo de un probador recesivo ( $g a l)$ y los dos probadores referidos previamente, homocigotos para Gal$S$. En ocasiones la mezcla de líneas hermanas y la formación de cruzas simples, triples o dobles de constitución alélica variable, pueden dificultar la clasificación de los materiales; en este trabajo, la línea LUG-357 proviene de una mezcla de líneas hermanas de apariencia fenotípica casi idéntica que provienen de varias autofecundaciones con constitución alélica diferente, y produjo una clasificación variable dentro de la misma. De acuerdo con los resultados, algunas plantas son homocigotas gal/gal y otras Ga1-m/Gal-m, como consecuencia de más de cinco ciclos de autofecundación y mantenimiento por pedigrí.

Los resultados respecto al locus Teosinte crossing $b a$ rrier-1 (tcb1) no permitieron detectar algún probador con las características establecidas anteriormente (Experimento 3). El Complejo de Incompatibilidad del Teocintle (TIC) funciona como una barrera eficiente siempre y cuando se encuentren al mismo tiempo el alelo $T c b 1-S$ y al menos un gene modificador ligado a los loci $g a 1$ y $t c b 1$ (Allen y Kermicle, 2001). Al parecer las versiones de W22 usadas en este estudio (W22-TIC V1.7 y W22-TIC V2.3) tienen una acción débil como barrera ya que durante los procesos de avance por autofecundación en Wisconsin, perdieron la estabilidad proporcionada por los genes modificadores (Stb) (Kermicle, 2006; Com. personal) ${ }^{1}$. Es necesario continuar los trabajos con las líneas apropiadas para este sistema de incompatibilidad, para poder discriminar correctamente los materiales de interés; en particular, las líneas usadas por Kermicle (2006). Asimismo, se esperaría clasificar correctamente los materiales deseados y avanzar en identificar la existencia de inhibidores o promotores para este sistema.

${ }^{1}$ Kermicle J L (2006) Laboratory of Genetics, University of Wisconsin, Madison, WI, 53706, USA.
Con base en lo anterior, es claro que la obtención de materiales comerciales o experimentales para usarse en pruebas de incompatibilidad requiere de varios años de estudio. Es necesario avanzar en la identificación de marcadores moleculares ligados a los factores de interés, con la capacidad de identificar los diferentes alelos y regiones del genoma involucrados en la incompatibilidad.

\section{CONCLUSIONES}

Se identificaron dos cruzas simples probadoras (NC354 x W22-P4830 y NC354 x W22-P5270) para el factor gametofitico-1 (gal) con el alelo Gal-S, con alta capacidad de discriminación y estabilidad. Las accesiones de las razas de maíz Dulce, Dulcillo del Noroeste y Reventador que poseen alelos $G a 1-S$, permitieron la identificación de probadores para el factor gametofitico-1.

La determinación de la constitución alélica de las líneas de la UDG LUG-03 y LUG-282 (Gal-m/Gal-m), LUG-14, LUG-358, LUG-379 y LUG-17L (gal/gal) permitirá obtener probadores con mejor adaptación a las condiciones subtropicales al combinarse con las líneas templadas $(T c b-S)$.

Ninguna de las cruzas simples entre líneas adaptadas y líneas templadas con el alelo $T c b-S$, fueron adecuadas como probadores para el Complejo de Incompatibilidad del Teocintle.

Es necesario intensificar los estudios del locus $t c b 1$, obtener líneas adaptadas a las condiciones de México con los diferentes sistemas de incompatibilidad conocidos en el género Zea, y tratar de incorporar el uso de marcadores moleculares para complementar los estudios actuales.

\section{AGRADECIMIENTOS}

A los Dres. J. Kermicle, M. M. Goodman y S. Taba por haber proporcionado parte del material genético usado en este estudio. A Pioneer Hi-Bred International y en especial al Dr. Baltazar Baltazar Montes, por las facilidades para incrementar algunos materiales en la estación experimental de Tapachula, Nay.

\section{BIBLIOGRAFÍA}

Evans M M S, J L Kermicle (2001) Teosinte crossing barrier-1, a locus governing hybridization of teosinte with maize. Theor. Appl. Genet. 103:259-265.

Hallauer A R (1975) Relation of gene action and type of tester in maize breeding procedures. Proc. 30th Annu. Corn Sorghum Res. Conf. pp:151-165.

Hallauer A R, J B Miranda Fo (1988) Quantitative Genetics in Maize Breeding. 2nd ed. Iowa State Univ. Press. Ames, IA. 468 p. 
Kermicle J L, J O Allen (1990) Cross-incompatibility between maize and teosinte. Maydica 35:399- 408 .

Kermicle J L (1997) Cross compatibility within the genus Zea. In: Gene Flow Among Maize Landraces, Improved Maize Varieties, and Teosinte: Implications for Transgenic Maize. J A Serratos, M C Willcox, F. Castillo (eds).CIMMYT, México, D. F. pp:40-43.

Kermicle J L (2006) A selfish gene governing pollen-pistil compatibility confers reproductive isolation between maize relatives. Genetics 172:499-506.

Kermicle J L, S Taba, M M S Evans (2006) The Gametophyte-1 locus and reproductive isolation among Zea mays subspecies. Maydica 51:1-8
Matzinger (1953) Comparison of three types of testers for the evaluation of inbred lines of corn. Agron. J. 45:493-495.

Nelson O E (1952) Non - reciprocal cross-sterility in maize. Genetics 37:101-124.

Nelson O E (1996) The gametophyte factors of maize. In: The Maize Handbook. M Freeling, V Walbot (eds). Springer-Verlag. pp:496-503.

Rawlings J O, D L Thompson (1962) Performance level as criterion for the choice of maize testers. Crop Sci. 2:217-220.

SAS Institute, Inc. (1992) SAS Technical Report P-229. SAS/STAT Software: Changes and Enhancements. SAS Institute, Inc., Cary, NC. 